\title{
Nahaufnahmen
}

\section{Spiel mir das Lied vom Leben: Zum Tod des italienischen Komponisten Ennio Morricone}

Play Me the Song of Life: On the Death of the Italian Composer Ennio Morricone

\section{Wolfram Goertz*ab}

[a] Redaktion Kultur / Wissen, Rheinische Post Verlagsgesellschaft, Düsseldorf, Deutschland. [b] Interdisziplinäre Ambulanz für Musikermedizin, Universitätsklinikum Düsseldorf, Düsseldorf, Deutschland.

\section{Zusammenfassung}

Ob Spaghetti-Western oder Kammermusik: Der legendäre Komponist Ennio Morricone war Profi durch und durch. Ein Nachruf.

Schlüsselwörter: Filmmusik, Morricone, Nachruf

\section{Abstract}

Whether spaghetti westerns or chamber music: The legendary composer Ennio Morricone was a professional through and through. An obituary.

Keywords: film music, Morricone, obituary

Jahrbuch Musikpsychologie, 2020, Vol. 29, Artikel e82, https://doi.org/10.5964/jbdgm.2019v29.82

Publiziert (VoR): 2020-10-26.

*Korrespondenzanschrift: Redaktion Kultur / Wissen, Rheinische Post Verlagsgesellschaft mbH, Zülpicher Straße 10, 40196 Düsseldorf, Deutschland E-Mail: Wolfram.Goertz@rheinische-post.de

\footnotetext{
Dieser Open-Access-Artikel steht unter den Bedingungen einer Creative Commons Namensnennung 4.0 International Lizenz, CC BY 4.0 (https://creativecommons.org/licenses/by/4.0/deed.de). Diese erlaubt für beliebige Zwecke (auch kommerzielle) den Artikel zu verbreiten, in jedwedem Medium zu vervielfältigen, Abwandlungen und Bearbeitungen anzufertigen, unter der Voraussetzung, dass

der Originalartikel angemessen zitiert wird.
}

Jeder Mensch kennt den Beginn von Beethovens Klavierstück „Für Elise“. E-Dis-E-Dis-E, eine unverwüstliche Tonfolge, wie eine Schleife. Auch Ennio Morricone kannte sie - aus ihrer Melancholie und Unschuld machte er den Hauch des Todes. Diese Mundharmonika. Charles Bronson. „Spiel mir das Lied vom Tod“. So wird man als Komponist weltberühmt und im Schlepptau auch der Film.

Solche Tricks des Tonsatzes liebte der italienische Filmmusikkomponist Ennio Morricone. Er war selbst ein glorreicher Halunke, ein Profi, der wusste, wie es geht. Vor allem reizte er seinen Beethoven in Sergio Leones legendärem Western penetrant aus: Er dreht jene Halbtonschraube immer länger, immer tiefer ins Gehirn. EDis-E-Dis-E-Dis-E-Dis-E-Dis-E. Bis kein Widerstand mehr da ist. Die Szene im Film hat etwas Surreales, Sonnenflimmerndes, doch besitzt sie eine weitere Pointe. Die Schleife steht in der Tonart a-Moll. Und welches Intervall ist es noch mal von A zum Dis? Eine übermäßige Quarte, der sogenannte Tritonus, auch „diabolus in musica“ genannt. Das Lied vom Tod mit der Musik vom Teufel. 
Mit solchen Details hat Morricone gespielt. Er ging einem ja auch stets mit voller Absicht an die Nieren. Er drang zu den Bereichen im Gehirn vor, in denen die emotionale Wehrlosigkeit des Menschen sitzt. Er ließ Tränen wie ein Alchemist fließen, ließ den Herzschlag rattern, als pumpe er Adrenalin in unsere Adern, er zerrte an den Nerven, er hatte ein unfassbares Gespür für das Kino und seine Dramaturgie. Morricone war der Maestro des Augenblicks, und trotzdem hörte seine Musik die Dinge voraus, die geschehen würden. Seine Musik soufflierte dem Publikum: Hört her, ich weiß alles, ich kenne den Fortgang. Ich kenne sogar das Ende.

Das Kennzeichen ihrer Qualität ist, dass fast jede Morricone-Musik auch ohne Film funktionieren kann. Kaum zu glauben? Nun, dann muss man sich nur bei YouTube ein von ihm dirigiertes Konzert anhören, bei dem unter anderem seine Musik zu Giuseppe Tornatores Film „Cinema Paradiso“ erklingt. Eine himmlisch schöne, wie von Gott für uns Sünder komponierte Musik ist das. Anfangs stimmt sie das Klavier als einsame Ballade an, in fast scheuem B-Dur. Dann legt das Orchester dicke Stricke ums Herz des Hörers und zieht langsam zu. "Cinema Paradiso“ ist eine der schönsten Liebeserklärungen ans Kino - Morricone sorgte dafür, dass man den Film auch im Ohr behielt.

Die Regisseure wussten, wie wichtig Morricone für ihre Arbeit war. Zu ihm kamen alle, die einen unverwechselbaren Sound benötigten: Sergio Leone, Bernardo Bertolucci, Pier Paolo Pasolini, Henri Verneuil, Roman Polánski, Pedro Almodóvar, Franco Zeffirelli, Mike Nichols, Oliver Stone, Quentin Tarantino. Für über 500 Filme hat er komponiert, Giuseppe Tornatore war zweifellos sein Lieblingsregisseur (neben Leone) - und er war 2016 in „La corrispondenza“ der letzte Filmmacher, mit dem Morricone gearbeitet hatte, da war Morricone bereits 88 Jahre alt. Er konnte einfach nicht aufhören, ihm fiel ja auch immer etwas ein.

Manchmal war er auftragsbedingt nur ein Raubkopierer, Bertolucci wollte das für seinen Film „1900“ so haben, weswegen Morricone etliche Melodien von Giuseppe Verdi eindickte und dann mit neuem Geschmack durch den orchestralen Entsafter schickte. Und bei Oliver Stones wüstem Thriller „U-Turn - Kein Weg zurück“ begriff Morricone schnell, dass der Film eine Odyssee durch die Kinogeschichte war, da ließ er sich zitattechnisch dann auch nicht lumpen. Morricone sagte einmal, als Komponist fürs Kino müsse man wie ein Chamäleon sein. Stimmt schon, trotzdem erkennt man seinen Stil immer. Wie er mit den Streichern dicke Teppiche auslegt. Wie er die Oboe herzerwärmend näseln lässt. Wie er das Blech vor dem Plärren bewahrt. Und wie er Instrumente mit magischer Bedeutung auflädt, etwa die Panflöte in „Es war einmal in Amerika“.

Morricone hat allerdings viel mehr komponiert als seine ungemein vielgestaltige Filmmusik, beispielsweise eine Chorkomposition zum 11. September, „Voci dal silenzio“, außerdem sinfonische Musik und Kammermusik. Tatsächlich wollte er anfangs einfach nur Komponist werden. In Goffredo Petrassi hatte er einen hochrangigen Lehrer, mit seinen Freunden und Kollegen Luigi Nono, Luigi Dallapiccola, Luciano Berio oder Franco Donatoni - allesamt sogenannte Neutöner - stand er oft gemeinsam auf Konzertplakaten.

Ihre Arbeit schätzte er sehr, wusste aber, dass jedes Publikum der Welt mit den Füßen abstimmen würde. Nonos Chormusik fand er großartig, aber in einem ZEIT-Interview erklärte Morricone einmal, warum sie zwischen Turin und Palermo kaum Publikum fand: "Die Italiener mögen zeitgenössische Musik nicht. Sie ist den Leuten hier ziemlich egal. Die wenigen Konzerte, die es gibt, finden vor leeren Reihen statt. Und wenn Sie innerhalb eines klassischen Konzerts mit Mozart und Beethoven etwas Modernes bringen, müssen Sie damit rechnen, dass das Publikum den Saal verlässt." 
In seinen ersten Jahren hatte er das selbst ausprobiert. 1958 war er sogar Besucher der Darmstädter Ferienkurse gewesen, eines Hotspots der Moderne. Doch später begriff er die Mechanismen des Betriebs. Neun Monate hatte er über seinem ersten Orchesterstück gesessen, für das Festival von Venedig. Als Honorar bekam er 60.000 Lire, damals ein Taschengeld. Von solchen Kompositionen konnte er keine Familie ernähren.

1946 hatte er begonnen, Arrangements für das Varietétheater zu schreiben, dann für das Radio und das Fernsehen. 1961 kam der erste Kinofilm, „Il federale“ von Luciano Salce. Viele Jahre war er mit dem Kino so beschäftigt, dass er seine ursprüngliche Kompositionsarbeit liegen ließ. In den Achtzigerjahren begann er wieder damit: "Ich selbst betrachte mich als einen Komponisten, der auf zwei Säulen steht." Maßlos ärgerte es ihn, dass er oft nur mit den italoamerikanischen „Spaghetti-Western" in Verbindung gebracht wurde.

Zwei Oscars bekam er, einen für sein Lebenswerk, 2016 einen weiteren für die Musik zu Tarantinos Western „The Hateful Eight“. Zuvor hatte Morricone mehrere Jahrzehnte lang keine Musik zu einem Western mehr geschrieben. Egal, Tarantino fehlte inm in der Sammlung, außerdem wusste er ja, wie es geht. Und als die Stelle mit dem Schnee kam, durch den der Weg ins Blutbad führt, da zahlte es sich mal wieder aus, dass Morricone, wie immer im guten Sinne altmodisch, seine Prinzipien der Spannungserzeugung nie verlernt hatte. Wiederholen. Insistieren. Schritt für Schritt stapfen. Und die Schraube wieder knirschen lassen.

Im Alter von 91 Jahren ist Ennio Morricone am 6. Juli 2020 in Rom gestorben. Die Mundharmonika zählte übrigens nicht zu seinen Lieblingsinstrumenten.

\section{Originalitätserklärung}

Der vorliegende Text wurde bereits am 8. Juli 2020 in DIE ZEIT Nr. 29/2020 veröffentlicht. 